\title{
Quarkyonic phase from quenched dynamical holographic QCD model
}

\author{
Xun Chen, ${ }^{a, b, 1}$ Danning $\mathbf{L i},{ }^{c, 2}$ Defu Hou ${ }^{a}$ and Mei Huang ${ }^{b, 3}$ \\ ${ }^{a}$ School of Nuclear Science and Technology, University of Chinese Academy of Sciences, \\ Beijing 100049, P.R. China \\ ${ }^{b}$ Central China Normal University, \\ Wuhan 430079, P.R. China \\ ${ }^{c}$ Department of Physics and Siyuan Laboratory, Jinan University, \\ Guangzhou 510632, P.R. China \\ E-mail: chenxun@ihep.ac.cn, lidanning@jnu.edu.cn, \\ houdf@mail.ccnu.edu.cn, huangmei@ucas.ac.cn
}

Abstract: Chiral and deconfinement phase transitions at finite temperature $T$ and quark number chemical potential $\mu$ are simultaneously studied in the quenched dynamical holographic QCD model within the Einstein-Dilaton-Maxwell framework. By calculating the corresponding order parameters, i.e., the chiral condensate and Polyakov loop, it is shown that the transition lines of these two phase transitions are separated in the $T-\mu$ plane. The deconfinement phase transition is shown to be always of crossover type and the transition line depends weakly on the baryon number density. Differently, the chiral transition is of crossover at small baryon number density and it turns to be of first order at sufficient large baryon number density. A critical endpoint (CEP), at which the transition becomes second order type, appears in the chiral transition line. This is the first time to realize the CEP of chiral phase transition in the $(T, \mu)$ plane using the holographic EMD (Einstein-MaxwellDilaton) model for two flavour case. It is observed that between these two phase transition lines, there is a region with chiral symmetry restored and color degrees still confined, which could be considered as the quarkyonic phase. Qualitatively, this behavior is in consistent with the result in the Polyakov-loop improved Nambu-Jona-Lasinio (PNJL) model.

KEYworDs: Gauge-gravity correspondence, Holography and quark-gluon plasmas, AdS-CFT Correspondence

ARXIV EPRINT: 1908.02000

\footnotetext{
${ }^{1}$ First author.

${ }^{2}$ Co-corresponding author.

${ }^{3}$ Corresponding author.
} 


\section{Contents}

1 Introduction

2 Setup for the quenched dynamical holographic QCD model at finite baryon chemical potential

3 Deconfinement transition at finite baryon density

4 Chiral phase transition and the quarkyonic phase 8

4.1 Building $V\left(\chi, F^{2}\right) \quad 9$

4.2 Interplay of chiral and deconfinement phase transition: quarkyonic phase 14

5 Conclusion

\section{Introduction}

Quantum Chromodynamics (QCD) is widely accepted as the fundamental theory of the strong interaction with two most important properties in the vacuum, i.e., the spontaneous chiral symmetry breaking and color confinement. At sufficient high temperature or/and baryon chemical potential, it is believed that the system will undergo phase transitions, involving the restoration of chiral symmetry and the release of color degrees of freedom. The interplay of chiral symmetry breaking and color confinement as well as the relation between chiral and deconfinement phase transitions at finite temperature and density reveal the fundamental property of quark dynamics and gluon dynamics, thus it attracts continuous interests [1-7]. In the limit of large number of colors $N_{c}$, the quarkyonic phase was expected [8-10] in certain baryon number density region, where the chiral symmetry is restored but color degrees of freedom are still confined.

It requires more efforts to understand the full properties of QCD, since chiral symmetry breaking and color confinement have a non-perturbative origin, when the traditional perturbative methods face enormous challenges. Lattice QCD, starting from the first principle at zero and small quark chemical potential [11-14], is regarded as an important tool to overcome the non-perturbative problem. Besides lattice QCD, other non-perturbative methods such as Dyson-Schwinger equations (DSEs) [15, 16], functional renormalization group equations (FRGs) [17-19] and QCD effective models have been developed. Among QCD low energy effective models, Nambu-Jona-Lasinio (NJL) model [20, 21] offers the mechanism of spontaneous chiral symmetry breaking and has been widely used in describing chiral phase transition and investigating QCD phase structures under variant extreme conditions. In this model, the QCD gluon-mediated interactions are replaced by effective interactions among quarks, which are built according to the global symmetries of QCD. 
The NJL model does not contain dynamical gluons, which can be improved by adding the Ginzburg-Landau type potential of the traced Polyakov loop to the lagrangian to describe gluon dynamics and an interaction term of the Polyakov loop with the quarks. The improved model is usually named the Polyakov-loop improved Nambu-Jona-Lasinio (PNJL) model [22-38].

In recent decades, the discovery of the anti-de Sitter/conformal field theory (AdS/CFT) correspondence and the conjecture of the gauge/gravity duality [39-41] leads a new way to solve the strong coupling problem of gauge theory. Comparing with the original AdS/CFT correspondence, it is necessary to break the conformal symmetry at low energy to describe QCD. Many efforts have been made towards more realistic holographic description of low energy phenomena of QCD in hadron physics [42-57] and hot/dense QCD [58-72], including the top-down approaches and bottom-up approaches (see [73-77]for reviews). The deconfinement phase transition [78-100] has been widely discussed with the expectation value of Polyakov loop is the order parameter of deconfinement phase transition. However, till recently, the dynamical spontaneous chiral symmetry breaking and chiral phase transition have been realized [101-108] with the chiral condensate as the order parameter.

In large $N_{c}$ limit, it has been proposed that the chiral symmetry and deconfinement phase transition can be splitting and there would be a quarkyonic phase [8], where the chiral symmetry is restored but still in confinement. The free energy of a heavy test quark added to the system is $e^{-\beta F_{q}}=\frac{1}{N_{c}}<L>$ and the baryon number density is $<N_{B}>\sim e^{-\beta\left(M_{B}-\mu_{B}\right)}$ with $\beta$ the inverse of temperature. The deconfinement temperature is around $T_{d} \sim \Lambda_{Q C D}$. When the baryon number chemical potential is small compared to the baryon mass, i.e. $\mu_{B} \ll M_{B}$, the number of baryons $<N_{B}>\sim e^{-\kappa_{1} N_{c}}$ tends to zero at large $N_{c}$ for temperatures of $T \sim \Lambda_{Q C D}$ and with $M_{B} \sim N_{c}$. Here, $\kappa_{1}$ is a number of order one. When $\mu_{B} \geq M_{B}$, the baryons begin to populate the system and the baryon number density becomes nonvanishing. Ref. [8] argues that there are at least three phases in the QCD phase diagram at large $N_{c}$ : the deconfinement phase with $T>T_{d}$, and in the region of $T<T_{d}$, there will be the mesonic phase which is confined and has vanishing baryon number density and the quarkyonic phase which has finite baryon number density and is confined [10]. However, in the real QCD case with $N_{c}=3$, we normally call the chiral symmetric and confined phase as the quarkyonic phase as shown in the PNJL model [10, 109].

In this paper, we make a step towards investigating the interplay between the deconfinement phase transition and chiral phase transition in a quenched dynamical holographic QCD model $[55,56,72]$. In this quenched dynamical holographic QCD model, the dilaton background describes the gluodynamics and the flavor/meson background describes the chiral dynamics, respectively, thus one can simultaneously realize the confinement/deconfinement phase transition and chiral symmetry breaking/restoration phase transition at finite temperature and chemical potential. However, it is worthy of mentioning that in the quenched dynamical holographic QCD model, the flavor background is added on the dilaton background as a probe, and the full QCD dynamics including the backreaction from the flavor background on the dilaton background or gluodynamics background has not been self-consistently solved yet, which is left for future work. 
To extend the quenched dynamical holographic QCD model to finite chemical potential, the quark chemical potential is introduced by a U(1) field in the Einstein-Dilaton-Maxwell framework. Except to fix the chemical potential dependence of the flavor background, one has also to fix the chemical potential dependence of the dilaton/gluodynamics potential, which can be determined by higher order baryon number fluctuations especially the kurtosis of baryon number fluctuations. From the experience in the PNJL model [110] as well as in the holographic QCD model [111], the kurtosis of baryon number fluctuations is dominated by contribution from gluodynamics. Therefore, we fix the chemical potential dependence of the dilaton field by fitting the lattice results of the kurtosis of baryon number fluctuations [112] at zero chemical potential. It is found that the deconfinement phase transition in the $(T, \mu)$ plane is always a crossover, this is in agreement with the result in the PNJL model [110]. By adding the coupling of $\chi^{6}$ with chemical potential, the critical end point (CEP) shows up along the chiral phase transition line. It is also found that the chiral symmetry restoration and deconfinement phase transitions are separated. More interestedly, it is observed that there exists a region where chiral symmetry is restored but color degrees of freedom are still confined. This is similar to the quarkyonic phase obtained in the PNJL model [110]. The possible reason for the separation of the chiral and deconfinement phase transitions is due to the quenched gluodynamical background, where the flavor dynamics is added as a probe.

The remainder of the paper is organized as follows: we give a review on the quenched dynamical holographic QCD model in section 2. In section 3, we fix the chemical potential dependence of the dilaton potential which describes gluodynamics through the baryon number susceptibilities and investigate the deconfinement phase transition in the $(T, \mu)$ plane. In section 4 we investigate the chiral phase transition and the phase diagram in the quenched dynamical holographic QCD model. In section 5, a brief summary is given.

\section{Setup for the quenched dynamical holographic QCD model at finite baryon chemical potential}

The deconfinement phase transition has been widely investigated in bottom-up holographic models [78-98]. Besides, it is possible to describe the chiral symmetry breaking and its restoration in the soft-wall model [101-103]. In this work, we investigate the interplay between the chiral and deconfinement phase transitions by using the dynamical holographic QCD model $[55-57,72,113,114]$. We emphasize that though the original AdS/CFT correspondence is in the large $N_{c}$ limit, the holographic QCD model is trying to describes phenomenology of real QCD in the case of $N_{c}=3$. In some sense, this is under a more general holographic conjecture for gauge/gravity duality $[115,116]$.

The full QCD contains quark dynamics and gluodynamics, and it is known that light flavor quark dynamics are responsible for the spontaneous chiral symmetry breaking, and gluodynamics are responsible for the color confinement. The dynamical holographic QCD model is constructed in the graviton-dilaton-scalar framework with the dilaton field and scalar field responsible for the gluodynamics and chiral dynamics, respectively. This dynamical holographic QCD model naturally resembles the renormalization group from ultra- 
violet (UV) to infrared (IR): at UV boundary, the theory goes to the limit of AdS/CFT, and the 5-dimension (5D) field in the bulk and 4D operator obeys the principle of AdS/CFT correspondence [42], and the model at IR is determined by QCD non-perturbative properties such as chiral condensate and gluon condensate or glueball properties. In [55-57, 71, 72], we see that the graviton-dilaton system can describe the pure gluon system including the glueball spectra, thermodynamical properties as well as transport properties quite well. After adding the flavor background and solving the deformed metric self-consistently, the total dynamical system can describe the meson spectra very well and the results are in agreement with experimental data [56].

However, it is not an easy task to solve the full system at finite temperature and chemical potential. Therefore, in this work, we use the quenched dynamical model with the flavor background added on the dilaton background as a probe. The chiral and deconfinement phase transitions at finite temperature in the quenched dynamical holographic QCD model has been investigated in ref. [104]. Here, we extend this scenario to finite chemical potential case, and try to study chiral and deconfinement phase transition in the $T-\mu$ plane.

To extend the quenched dynamical holographic QCD model in refs. [55, 56, 72] to finite chemical potential, we introduce an extra U(1) field in the Einstein-Dilaton-Maxwell framework, and the action in the string frame takes the form of:

$$
\begin{aligned}
S_{\text {total }}^{s} & =S_{G}^{s}+S_{M}^{s}, \\
S_{G}^{s} & =\frac{1}{16 \pi G_{5}} \int d^{5} x \sqrt{-g^{s}} e^{-2 \Phi}\left[R^{s}+4 \partial_{\mu} \Phi \partial^{\mu} \Phi-V_{G}^{s}(\Phi)-\frac{h(\Phi)}{4} e^{\frac{4 \Phi}{3}} F_{\mu \nu} F^{\mu \nu}\right], \\
S_{M}^{s} & =-\int d^{5} x \sqrt{-g^{s}} e^{-\Phi} \operatorname{Tr}\left[\nabla_{\mu} X^{\dagger} \nabla^{\mu} X+V_{X}^{s}\left(|X|, F_{\mu \nu} F^{\mu \nu}\right)\right] .
\end{aligned}
$$

Here $S_{\text {total }}$ is the full 5D action, $S_{G}$ is the $5 \mathrm{D}$ action for dilaton background describing gluodynamics, and $S_{M}$ is the 5D action for matter sector describing chiral dynamics, respectively. The lower-case $s$ represents the string frame, $g^{s}$ is the determinant of metric $g_{\mu \nu}, G_{5}$ is the $5 \mathrm{D}$ Newton constant, $\Phi$ is the dilaton field, and $X$ is the bulk scalar field which corresponds to $\bar{q} q$ condensate of QCD. $V_{G}$ represents the dilaton potential, and $V_{X}$ is the bulk scalar potential coupled with the strength tensor of gauge field. The leading term in $V_{X}$ is the mass term $m_{5}^{2} X X^{\dagger}$, which can be determined as $m_{5}^{2}=-3$ from the AdS/CFT prescription $m_{5}^{2}=(\Delta-p)(\Delta+p-4)$ by taking $\Delta=3, p=0[41,79] . h(\Phi)$ is a gauge kinetic function constraining the $\mu$ dependence of the system and will be fixed by fitting the lattice data on baryon number susceptibilities. $F_{\mu \nu}$ are the strength tensor of gauge field dual to the baryon number current. If $F_{\mu \nu}=0$, the system is reduced to zero chemical potential case, and $F_{\mu \nu} \neq 0$ corresponds to finite baryon number chemical potential case of the system.

To consider the gravity dual of QCD at finite temperature and baryon number density, we can take the following metric ansatz in the string frame:

$$
d s^{2}=\frac{e^{2 A_{s}(z)}}{z^{2}}\left[-f(z) d t^{2}+\frac{1}{f(z)} d z^{2}+d \vec{x}^{2}\right] .
$$

As discussed in ref. [93], it is more convenient to work out thermodynamics in the Einstein frame, therefore we transform the action into Einstein frame by a conformal transformation 
of metric:

$$
g_{\mu \nu}^{s}=e^{\frac{4 \Phi}{3}} g_{\mu \nu}^{e},
$$

then the action of the dilaton background part becomes:

$$
S_{G}^{e}=\frac{1}{16 \pi G_{5}} \int d^{5} x \sqrt{-g^{e}}\left[R^{e}-\frac{4}{3} \partial_{\mu} \Phi \partial^{\mu} \Phi-V^{e}(\Phi)-\frac{h(\Phi)}{4} F_{\mu \nu} F^{\mu \nu}\right],
$$

with

$$
V^{e}(\Phi)=e^{4 / 3 \Phi} V^{s}(\Phi) .
$$

In the Einstein frame, the metric ansatz becomes:

$$
d s^{2}=\frac{e^{2 A_{e}(z)}}{z^{2}}\left[-f(z) d t^{2}+\frac{1}{f(z)} d z^{2}+d \vec{x}^{2}\right] .
$$

Here, the two metric warp factors in two frames follow the relationship of $A_{s}=A_{e}+\frac{2 \Phi}{3}$. When considering finite chemical potential, the only non-vanishing component of the gauge potential $A_{\mu}$ of the strength tensor $F_{\mu \nu}$ is the time component $A_{t}$, i.e. $A=A_{t} d t$.

Inserting the above ansatz, one can derive the following equations of motion after certain simplifications [104]:

$$
\begin{array}{r}
A_{e}^{\prime \prime}-A_{e}^{\prime 2}+\frac{2}{z} A_{e}^{\prime}+\frac{4 \Phi^{\prime 2}}{9}=0, \\
A_{t}^{\prime \prime}+\left(\frac{h^{\prime}}{h}+A_{e}^{\prime}-\frac{1}{z}\right) A_{t}^{\prime}=0, \\
f^{\prime \prime}+\left(3 A_{e}^{\prime}-\frac{3}{z}\right) f^{\prime}-e^{-2 A_{e}} z^{2} h(\Phi) A_{t}^{\prime 2}=0 .
\end{array}
$$

The Hawking temperature of the black hole solution can be evaluated numerically by

$$
T=\frac{f^{\prime}\left(z_{h}\right)}{4 \pi} .
$$

Following [56], we take the dilaton field in the form of

$$
\Phi(z)=\alpha \tanh \left(\beta^{2} z^{2}+\gamma^{4} z^{4}\right)
$$

which tends to $z^{2}$ power thus goes to the $\mathrm{AdS}_{5}$ limit at the UV boundary and approaches a positive constant for a possible crossover transition at IR. Then one can solve eqs. (2.9)(2.11) by imposing the following boundary conditions at the boundary $z=0$ and the horizon $z=z_{h}$ :

$$
\begin{aligned}
A_{t}\left(z_{h}\right) & =f\left(z_{h}\right)=0 \\
f(0) & =1 \\
A_{t}(0) & =\mu+\rho z^{2}+\ldots
\end{aligned}
$$

Here, $\mu$ is the quark chemical potential and $\rho$ is quark number density. 
Following the procedure in ref. [93], we can calculate the pressure of the system through the entropy density

$$
s=\frac{e^{3 A\left(z_{h}\right)}}{4 z_{h}^{3}} .
$$

For fixed values of the chemical potential, the pressure density can be calculated by the integral

$$
p=\int[s d T+\rho d \mu]=\int\left[s\left(\frac{\partial T}{\partial z_{h}} d z_{h}+\frac{\partial T}{\partial \mu} d \mu\right)+\rho d \mu\right]
$$

and the energy density of the system can be derived:

$$
\epsilon=-p+s T+\mu \rho
$$

At zero chemical potential $\mu=0$, by fitting the lattice results of equation of state for $N_{f}=2$ QCD [117], one can fix parameters $\alpha=1.8, \beta=0.4 \mathrm{GeV}, \gamma=0.42 \mathrm{GeV}$ as in ref. [104]. With these parameters, the pseudo critical temperature for the crossover is around $T_{0}=217 \mathrm{MeV}[104]$.

In order to describe the system at finite chemical potential, we also need to fix $h(\Phi)$ in eq. (2.2) and (2.6), which describes the chemical potential dependence of the gluondynamical potential of the system. From the experience in the PNJL model [110], we can use the higher order baryon number fluctuations especially the ratio of fourth over second order cumulants of baryon number fluctuations to determine $h(\Phi)$. The kurtosis of baryon number fluctuations is given by $\kappa \sigma^{2}=C_{4}^{B} / C_{2}^{B}$ with the variance $\sigma^{2}=C_{2}^{B}$ and the kurtosis $\kappa=C_{4}^{B} /\left(\sigma^{2}\right)^{2}$, and the cumulants of baryon number distributions are given by $C_{n}^{B}=V T^{3} \chi_{n}^{B}$, where the baryon number susceptibilities are defined as:

$$
\chi_{n}^{B}=\frac{\partial^{n}\left[P / T^{4}\right]}{\partial\left[\mu_{B} / T\right]^{n}}
$$

and $P, V$ are the pressure and volume of the system, and $\mu_{B}=3 \mu$ is the baryon number chemical potential. The calculation of baryon number fluctuations in the holographic QCD model of [96] has been investigated in ref. [111]. The baryon number fluctuations in the PNJL model has been investigated in ref. [110] and it is observed that in the PNJL model [110] that the ratio of fourth over second order cumulants of net-baryon number fluctuations $\kappa \sigma^{2}$ at zero baryon number density is dominated by contribution from gluodynamics. Therefore, we fix the chemical potential dependence of the dilaton field by fitting the lattice results of the kurtosis of baryon number fluctuations [112] at zero chemical potential.

The kurtosis $\kappa \sigma^{2}$ as a function of the normalized temperature $T / T_{0}$ with $T_{0}=217 \mathrm{MeV}$ comparing with lattice result [112] is shown in figure 1 . It is found that when $h(\Phi)=\frac{7}{10}$, the result of kurtosis from the dilaton background, i.e., from the gluodynamical contribution in the quenched dynamical holographic QCD model is in good agreement with lattice results.

With the setup in this section, we are ready to investigate the deconfinement and chiral phase transitions in the quenched dynamical holographic QCD model at finite temperature as well as finite chemical potential. 


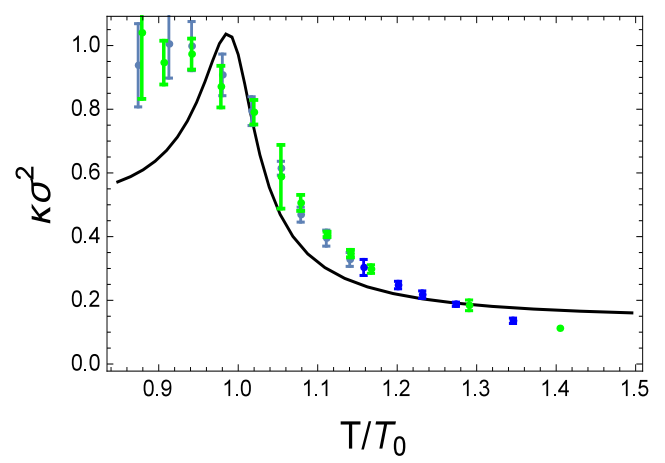

Figure 1. $\kappa \sigma^{2}$ as functions of the normalized temperature $T / T_{0}$ with $T_{0}=217 \mathrm{MeV}$ the pseudo critical temperature at zero chemical potential $\mu=0$. The black line is for $h(\Phi)=7 / 10$ which is in agreement with lattice results in ref. [112].

\section{Deconfinement transition at finite baryon density}

In order to investigate the deconfinement phase transition, the expectation value of the Polyakov loop is often used as an order parameter, which is defined as

$$
L(T)=\frac{1}{N_{c}} \operatorname{Tr} \mathrm{P} \exp \left(i g \int_{0}^{\frac{1}{T}} \hat{A_{0}}(\tau, \vec{x}) d \tau\right) .
$$

Here $N_{c}$ is the color number, $\mathrm{P}$ indicates path ordering, $\mathrm{g}$ is the coupling, the trace $\mathrm{Tr}$ is computed over the fundamental representation of $\mathrm{SU}\left(N_{c}\right)$ and $\hat{A}_{0}$ is the non-Abelian gauge field potential operator. The expectation value $\langle L(T)\rangle$ vanishes in the confined phase guaranteed by the center symmetry, and it is nonzero $\langle L(T)\rangle \neq 0$ in the deconfined phase, which indicates the center symmetry is broken. From the holographic dictionary, the expectation value of the Polyakov loop is related to the Nambu-Goto action $S_{N G}$ for the string world sheet [41]

$$
S_{N G}=\frac{1}{2 \pi \alpha_{p}} \int d^{2} \eta \sqrt{\operatorname{det}\left(g_{\mu \nu}^{s} \mathcal{X}_{a}^{\mu} \mathcal{X}_{b}^{\nu}\right)}
$$

in the following way

$$
\langle L(T)\rangle=\int D \mathcal{X} e^{-S_{N G}}
$$

where $\frac{1}{2 \pi \alpha_{p}}$ is the string tension, $g_{\mu \nu}^{s}$ is the metric in the string frame, and $\mathcal{X}_{a}^{\mu}$ is the embedding function of the worldsheet in the target space, $\mu, \nu$ are the five dimensional space-time indices and $a, b$ represent the worldsheet coordinates. From the metric of (2.4), we have

$$
S_{N G}=\frac{1}{2 \pi \alpha_{p}} \int_{0}^{z_{h}} d z \frac{e^{2 A_{s}}}{z^{2}} \sqrt{1+f(z)\left(\vec{x}^{\prime}\right)^{2}},
$$

with $g_{p}=\frac{1}{2 \alpha_{p}}$ the redefinition of the string tension. The prime denotes the derivative with respect to $z$. Then, the equation of motion for $\vec{x}$ can be derived as

$$
\left[\frac{e^{2 A_{s}}}{z^{2}} f(z) \vec{x}^{\prime} / \sqrt{1+f(z)\left(\vec{x}^{\prime}\right)^{2}}\right]^{\prime}=0 .
$$




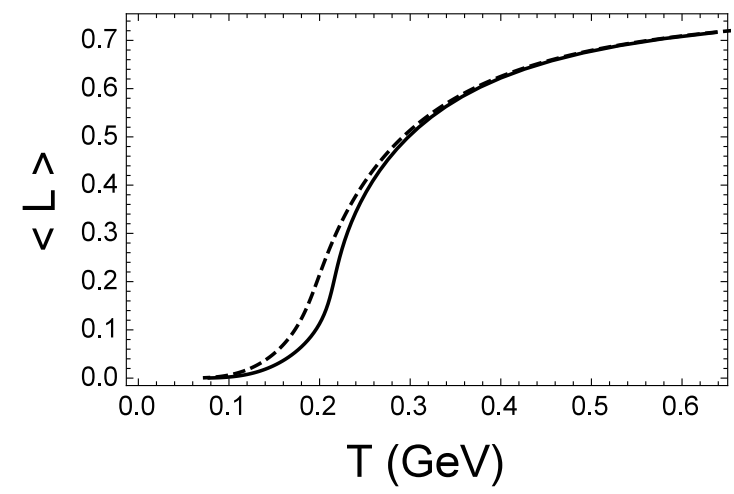

(a)

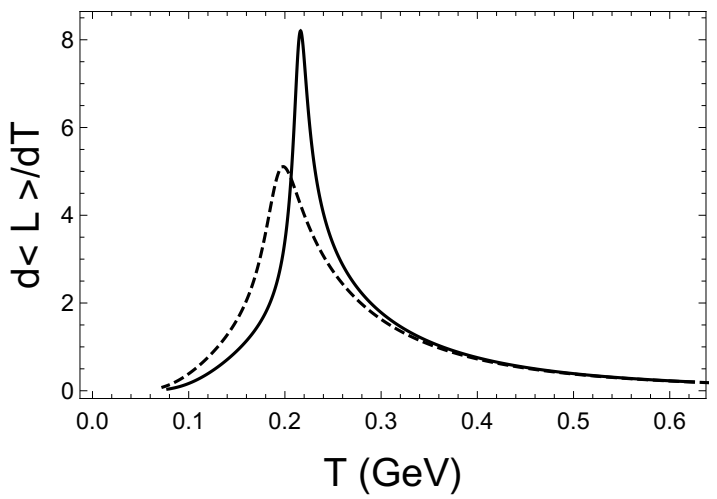

(b)

Figure 2. The expectation value of the Polyakov loop $\langle L\rangle$ (a) and its derivative $\frac{d<L\rangle}{d T}$ (b) as a function of the temperature in the cases of $\mu=0$ (solid line) and $\mu=0.5 \mathrm{GeV}$ (dashed line), respectively.

Substituting the $\vec{x}^{\prime}$ into the action $S_{N G}$, the minimal world sheet can be obtained as

$$
S_{0}=c_{p}+S_{0}^{\prime}=c_{p}+\frac{g_{p}}{\pi T} \int_{0}^{z_{h}} d z\left(\frac{e^{2 A_{s}}}{z^{2}}-\frac{1}{z^{2}}\right)
$$

where $c_{p}$ is a normalization constant. Finally, we can get the expectation value of the Polyakov loop:

$$
\langle L(T)\rangle=\omega e^{-S_{0}}=e^{C_{p}-S_{0}^{\prime}},
$$

with $C_{p}$ another normalization constant and $\omega$ a weight coefficient. By fitting two-flavor lattice results from [118], we take $C_{p}=-0.25, g_{p}=0.86$.

The expectation value for the Polyakov loop $\langle L\rangle$ at the chemical potentials $\mu=0$ and $\mu=0.5 \mathrm{GeV}$ are given in figure $2(\mathrm{a})$. We can see that at low temperature the system is in confined phase with vanishing $\langle L\rangle$, while at large temperature it tends to a constant showing a deconfinement phase transition. The transition from the confined phase to the deconfined phase is smooth, showing a crossover type transition. Usually, one can extract the corresponding pseudo critical temperatures through the derivative of $\frac{d\langle L\rangle}{d T}$, and the location of the peak gives the pseudo critical temperature. In figure 2(b), the results for $\frac{d\langle L\rangle}{d T}$ are given, which shows a weak dependence of critical temperature on the chemical potential. We then calculate the temperature dependent Polyakov loop up to the chemical potential $\mu=0.8 \mathrm{GeV}$, and obtain the $T-\mu$ phase diagram for deconfinement phase transition as shown in figure 3. From this figure, one can see that the deconfinement transition temperature is always a crossover and its critical temperature depends weakly on the chemical potential. This finding is consistent with that in the PNJL model [110].

\section{Chiral phase transition and the quarkyonic phase}

In previous sections, we have fixed the chemical potential dependence of the dilaton background from the equation of state and baryon number susceptibilities, and we have in- 


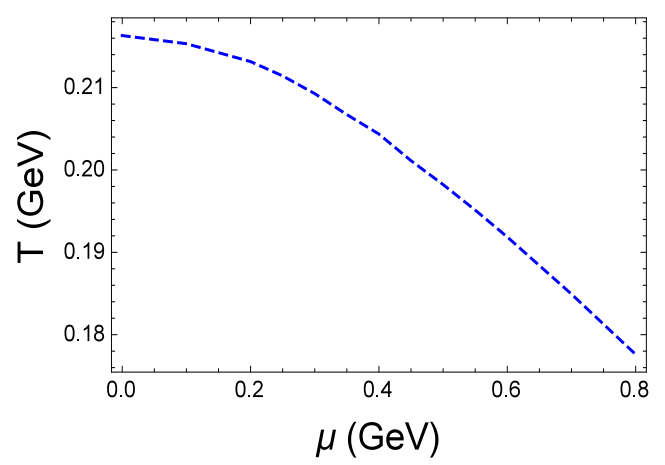

Figure 3. The deconfinement phase transition line in the $(T, \mu)$ plane in the quenched dynamical holographic QCD model.

vestigated the deconfinement phase transition in the $(T, \mu)$ plane. In this section, we will discuss the chiral phase transition at finite temperature and chemical potential.

\subsection{Building $V\left(\chi, F^{2}\right)$}

We will consider the action part in eq. (2.3), which includes the dynamics of the scalar field $X$, corresponding to the chiral condensate $\sigma \equiv\langle\bar{q} q\rangle$. When the scalar field $X$ obtains a vacuum expectation value $X_{0}$, the $\mathrm{SU}(2)_{L} \times \mathrm{SU}(2)_{R}$ symmetry of the matter sector $S_{M}$ would be broken. We consider the two-flavor case $N_{f}=2$ with $m_{u}=m_{d}$, and we set $X_{0}(z)=\chi(z) I_{2} / 2$ with $I_{2}$ the $2 \times 2$ identity matrix. From eq. (2.3), the action reduces to the form of

$$
S_{\chi}=-\int d^{5} x \sqrt{-g^{s}} e^{-\Phi}\left[\frac{1}{2} g^{z z} \chi^{\prime 2}+V\left(\chi, F^{2}\right)\right]
$$

where $V\left(\chi, F^{2}\right) \equiv \operatorname{Tr}\left(V_{X}\left(X, F^{2}\right)\right)$. The equation of motion can be easily derived as

$$
\chi^{\prime \prime}(z)+\left(-\frac{3}{z}+3 A_{s}^{\prime}(z)-\phi^{\prime}(z)+\frac{f^{\prime}(z)}{f(z)}\right) \chi^{\prime}(z)+\frac{e^{2 A_{s}(z)}}{z^{2} f(z)} \partial_{\chi} V\left(\chi(z), F^{2}\right)=0 .
$$

Generally, the potential $V_{X}$ could be of any form satisfying the $\mathrm{SU}(2)_{L} \times \mathrm{SU}(2)_{R}$ symmetry. In previous studies [102, 103], it is taken as a simple form $V_{X}\left(X, F^{2}\right)=$ $M_{5}^{2} X^{+} X+\alpha\left(X^{+} X\right)^{2}$ with $M_{5}^{2}=-3$ from the AdS/CFT prescription, or equivalently

$$
V\left(\chi, F^{2}\right)=-\frac{3}{2} \chi^{2}+v_{4} \chi^{4}
$$

It is shown that $v_{4}$ should be positive in order to have chiral symmetry breaking at low temperature in chiral limit. Therefore, firstly, we would have a test on this simple form of potential. Inserting this potential into eq. (4.2), one can obtain the leading $\operatorname{UV}(z \rightarrow 0)$ expansion of $\chi(z)$ as

$$
\chi(z)=m_{q} \zeta z+\ldots+\frac{\sigma}{\zeta} z^{3}+\ldots,
$$

where $\sigma$ is the chiral condensate (order parameter) and $\zeta=\frac{\sqrt{3}}{2 \pi}$ is the normalization constant obtain by matching correlation function $\langle\bar{q} q(p) \bar{q} q(0)\rangle$ with $4 \mathrm{D}$ calculation (for details, please refer to [119]). Near the horizon, the regular condition of $\chi$ requires 


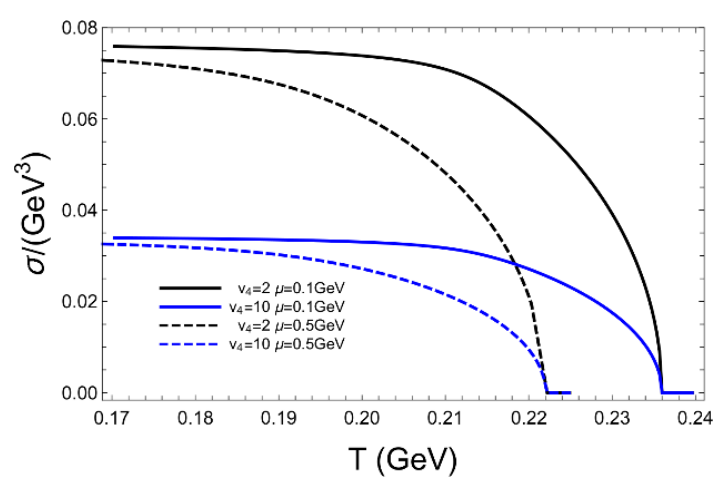

(a)

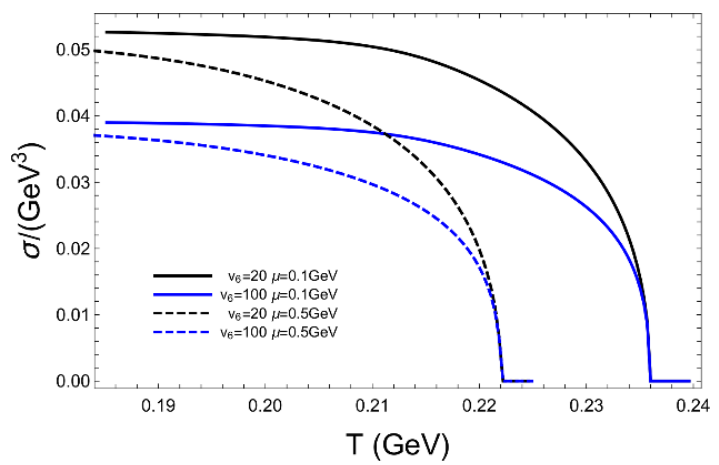

(b)

Figure 4. Chiral condensate $\sigma$ in chiral limit $\left(m_{q}=0\right)$ as a function of the temperature $\mathrm{T}$ for different potentials $V\left(\chi, F^{2}\right)$ and chemical potentials. Panel (a) gives the results for $V\left(\chi, F^{2}\right)=$ $-\frac{3}{2} \chi^{2}+v_{4} \chi^{4}$ when $v_{4}=2, \mu=0.1 \mathrm{GeV}$ (black solid line), $v_{4}=2, \mu=0.5 \mathrm{GeV}$ (black dashed line), $v_{4}=10, \mu=0.1 \mathrm{GeV}$ (blue solid line), $v_{4}=10, \mu=0.5 \mathrm{GeV}$ (blue dashed line). Panel (b) gives the results for $V\left(\chi, F^{2}\right)=-\frac{3}{2} \chi^{2}+v_{6} \chi^{6}$ when $v_{6}=20, \mu=0.1 \mathrm{GeV}$ (black solid line), $v_{6}=20$, $\mu=0.5 \mathrm{GeV}$ (black dashed line), $v_{6}=100, \mu=0.1 \mathrm{GeV}$ (blue solid line), $v_{6}=100, \mu=0.5 \mathrm{GeV}$ (blue dashed line).

$\frac{1}{f(z)}\left(f^{\prime} \chi^{\prime}+e^{2} A_{s} \partial_{\chi} V(\chi) / z^{2}\right)$ to be finite at $z=z_{h}$. Taking the metric solved in previous sections and imposing the above UV and IR boundary conditions, one can solve $\sigma$ with respect to $m_{q}, T$ and $\mu$.

Firstly we check whether the spontaneous symmetry breaking is described well. Therefore, we take $m_{q}=0$, i.e. in chiral limit. Then, we take different values of $v_{4}$ and $\mu$ and solve out $\sigma$ as a function of temperature in figure 4 (a) $\left(v_{4}=2, \mu=0.1 \mathrm{GeV}\right.$ (black solid line), $v_{4}=2, \mu=0.5 \mathrm{GeV}$ (black dashed line), $v_{4}=10, \mu=0.1 \mathrm{GeV}$ (blue solid line), $v_{4}=10, \mu=0.5 \mathrm{GeV}$ (blue dashed line)). From the figure, one could see that, for different values of $v_{4}$, the qualitative results for $\sigma$ are the same. At low temperature, chiral symmetry is broken by finite condensate $\sigma$, while above a certain temperature $T_{c}, \sigma$ turns to zero and chiral symmetry is restored. The phase transition is of second order type. It could also be seen that $T_{c}$ is not depend on $v_{4}$ for a fixed $\mu .{ }^{1}$ Also, we find that a larger $v_{4}$ leads to a smaller $\sigma$ at the same temperature when $v_{4}>0$, while there is no condensate when $v_{4} \leq 0$.

Up to finite temperature, it seems chiral phase transition could be well described by such a simple model. However, when one analyze the effect of baryon chemical potential $\mu$ one finds that the phase transition is always second order for any value of $\mu$. There is no first order transition region for any value of $\mu$. It is inconsistent with the expected phase diagram in $T-\mu$ plane for real QCD. Therefore, additional term in $V_{X}$ might be necessary. A direct extension is including the higher power $\left(X^{+} X\right)^{3}$ which satisfies the symmetry and considering effective potential

$$
V\left(\chi, F^{2}\right)=-\frac{3}{2} \chi^{2}+v_{4} \chi^{4}+v_{6} \chi^{6}
$$

\footnotetext{
${ }^{1}$ Actually, this could be easily understood by expanding eq. (4.2) to the leading order near $T_{c}$. (For details, see [120]).
} 


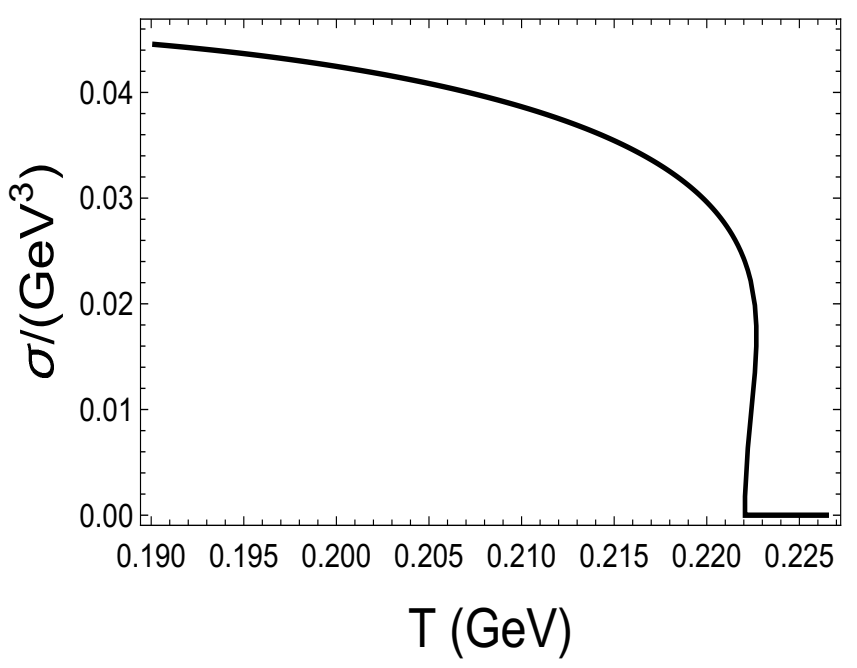

Figure 5. Chiral condensate $\sigma$ in chiral limit $\left(m_{q}=0\right)$ as a function of the temperature $\mathrm{T}$ for $V\left(\chi, F^{2}\right)=-\frac{3}{2} \chi^{2}+v_{4} \chi^{4}+v_{6} \chi^{6}$ with $v_{4}=-2, v_{6}=100$ and $\mu=0.5 \mathrm{GeV}$.

Similarly, we take chiral limit to study the phase structure first. We take $v_{4}=0$ and different values of $v_{6}=20, \mu=0.1 \mathrm{GeV}$ (black solid line), $v_{6}=20, \mu=0.5 \mathrm{GeV}$ (black dashed line), $v_{6}=100, \mu=0.1 \mathrm{GeV}$ (blue solid line), $v_{6}=100, \mu=0.5 \mathrm{GeV}$ (blue dashed line) in figure $4(\mathrm{~b})$. From the figure, we find that the effect of $v_{6}$ is very similar to $v_{4}$. It will not affect the location of $T_{c}$, while it suppresses chiral condensate for a given value of $T$ and $\mu$. The transition order of chiral transition is still kept as second order one for any value of $\mu$.

Then we turn on the effect of $v_{4}, v_{6}$ simultaneously, and we find that when both $v_{4}$ and $v_{6}$ are positive, the qualitative results are almost the same as the above two cases. But if $v_{4}$ become negative, the solution structure near the transition point where $\sigma$ turns to zero becomes different. As an example, we take $v_{4}=-2, v_{6}=100, \mu=0.5 \mathrm{GeV}$ and show the results in figure 5. In this situation, we could see that a triple-valued region of $\sigma$ appears in a narrow temperature region $0.222 \mathrm{GeV}<\mathrm{T}<0.223 \mathrm{GeV}$, which indicates a first order phase transition. Though the transition order depends only on the sign of $v_{4}$ and is independent on $\mu$, we see the possibility of constructing a model to describe different transition orders in different region of $\mu$ by changing the sign of the sub-leading power term $v_{4}$. If $v_{4}$ effectively changes its value from positive at small $\mu$ to negative at large $\mu$, one might expect a change of transition order. It is easy to see that if one adds a term like

$$
\alpha F^{2}\left(X^{+} X\right)^{2}
$$

to the action, effectively, the coefficient of $\chi^{4}$ term becomes

$$
-\lambda_{4} A_{t}^{\prime 2} \frac{z^{4}}{e^{4 A_{s}}}+v_{4}
$$

Here, we have inserted $A_{t}=A_{t}(z), A_{z}=0$ and $A_{i}=0$ for spatial components of the gauge potential. When $\mu=0$, we take $v_{4}>0$, the transition is second order; while when $\mu$ is sufficient large, $-\lambda_{4} A_{t}^{\prime 2} \frac{z^{4}}{e^{4 A_{s}}}+v_{4}$ will effectively become negative dominant at 


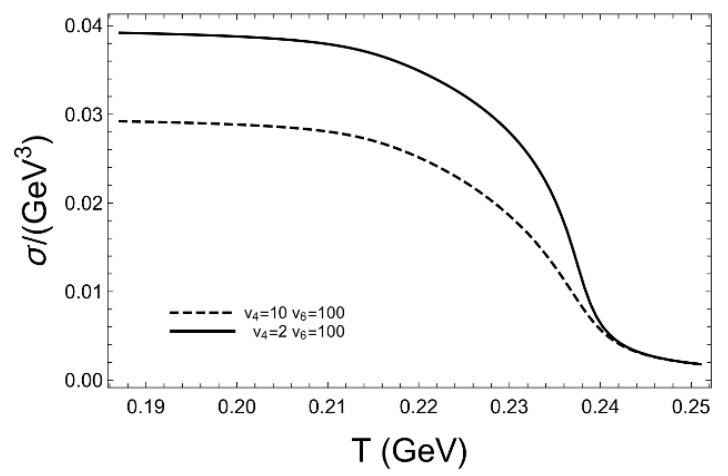

(a)

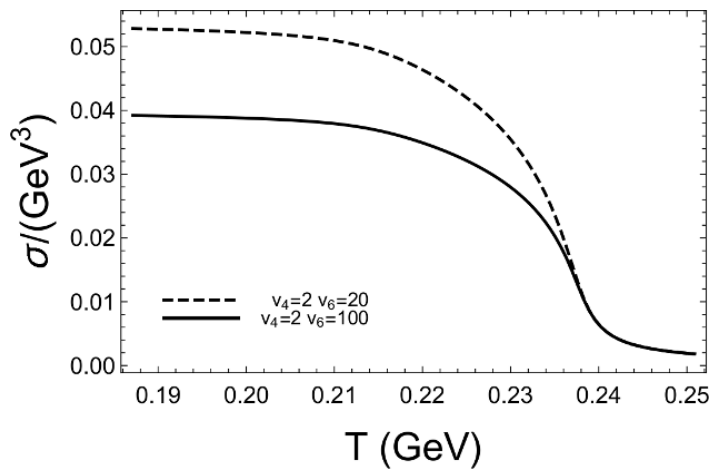

(b)

Figure 6. Chiral condensate $\sigma$ as a function of the temperature $\mathrm{T}$ for $\mu=0$ and $m_{q}=5 \mathrm{MeV}$, with $V\left(\chi, F^{2}\right)$ taking the form of eq. (4.8). Panel (a) gives results for $v_{4}=2, v_{6}=100$ (solid line) and $v_{4}=10, v_{6}=100$ (dashed line). Panel (b) gives results for $v_{4}=2, v_{6}=100$ (solid line) and $v_{4}=2, v_{6}=20$ (dashed line). Here, since $\mu=0$, the values of $\lambda_{2}, \lambda_{4}, \lambda_{6}$ will not affect $\sigma$.

certain region and the transition order might turn to first order. Of course, generally, we can consider any power obeying the symmetry. Here we only consider the first powers $F^{2}\left(X^{+} X\right), F^{2}\left(X^{+} X\right)^{2}, F^{2}\left(X^{+} X\right)^{3}$ and assume the effect of higher power term would be suppressed by certain mechanism.

According to the above discussion, we will consider the following general form of potential $V\left(\chi, F^{2}\right)$

$$
V\left(\chi, F^{2}\right)=\left(\frac{3}{2}+\lambda_{2} A_{t}^{\prime 2} \frac{z^{4}}{e^{4 A_{s}}}\right) \chi^{2}+\left(\lambda_{4} A_{t}^{\prime 2} \frac{z^{4}}{e^{4 A_{s}}}-v_{4}\right) \chi^{4}+\left(\lambda_{6} A_{t}^{\prime 2} \frac{z^{4}}{e^{4 A_{s}}}-v_{6}\right) \chi^{6} .
$$

Here, $v_{4}, v_{6}$ terms are corresponding to $\left(X^{+} X\right)^{2},\left(X^{+} X\right)^{3}$ term, $\lambda_{2}, \lambda_{4}, \lambda_{6}$ terms are corresponding to $F^{2} X^{+} X, F^{2}\left(X^{+} X\right)^{2}, F^{2}\left(X^{+} X\right)^{3}$ terms. We have imposed that at finite $\mu$ only $A_{t}(z)$ is non-vanishing and replaced $F^{2}$ with it.

Therefore, up to now, from the analysis in chiral limit, we have introduced five parameters $v_{4}, v_{6}$ and $\lambda_{2}, \lambda_{4}, \lambda_{6}$ to describe chiral phase transition. Now we can start to describe the phenomenology of real QCD. So in the rest part we will take $m_{q}=0.005 \mathrm{GeV}$, close to the physical value of $u, d$ quarks. Note that when $\mu=0, A_{t} \equiv 0$ and $\lambda_{2}, \lambda_{4}, \lambda_{6}$ will not enter the equation of motion of $\chi$. In this situation, only $v_{4}, v_{6}$ affect the behavior of $\sigma$. In figure 6 , we show the effects of $v_{4}, v_{6}$ on $\sigma$ when $\mu=0$. From the figure, we could see that, since $m_{q} \neq 0$, the phase transition turns from second order to crossover type. Different from chiral limit, $\sigma$ approaches to zero at high temperature instead of being exactly zero at $T_{c}$. Here, we simply define the pseudo transition temperature $T_{c}$ as $\left.\frac{\partial^{2} \sigma}{\partial^{2} T}\right|_{T_{c}}=0$. From the figure, we can see that the larger value of $v_{4}$ or $v_{6}$ leads to a smaller value of $\sigma$. Also, they would change the pseudo transition temperature. Thus, changing $v_{4}, v_{6}$ we can get different values of vacuum condensate $\sigma_{0} \equiv \sigma(T=0, \mu=0)$ and $T_{c}$. Therefore, by fitting $\sigma=(0.340 \mathrm{GeV})^{3}, \mathrm{~T}_{\mathrm{c}}=0.238 \mathrm{GeV}$, close to the results with vanishing $\mu$ from previous studies $[10,118]$, we can fix $v_{4}=2, v_{6}=100$ firstly. Then, only three free parameters $\lambda_{2}, \lambda_{4}, \lambda_{6}$ are left. 


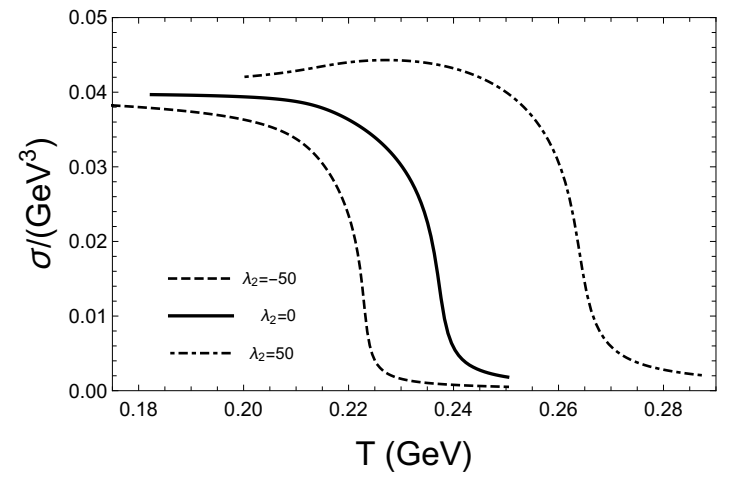

(a)

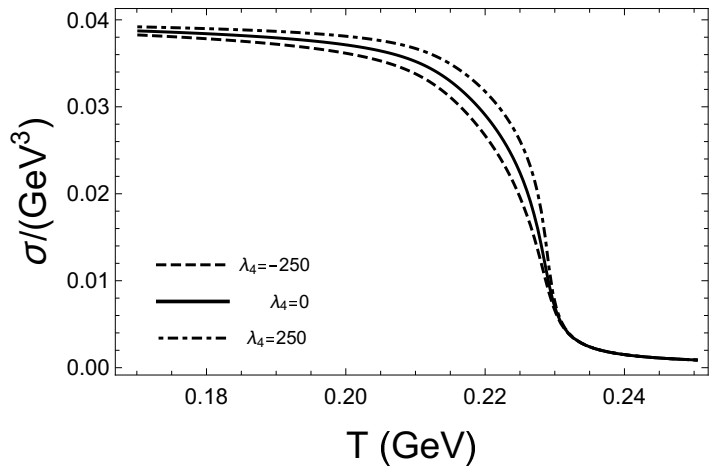

(b)

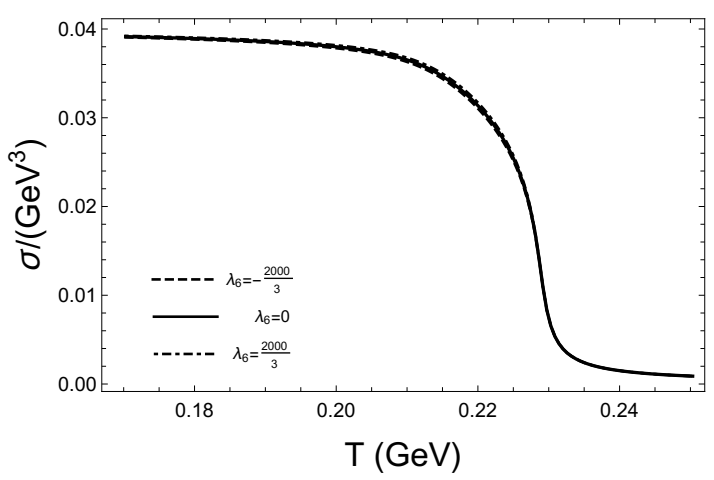

(c)

Figure 7. The chiral condensate $\sigma$ as a function of $\mathrm{T}$ for different parameters at $\mu=0.1 \mathrm{GeV}$. (a) The solid line is for $\lambda_{2}=0$, the dashed line is for $\lambda_{2}=-50$ and the dot-dashed line is for $\lambda_{2}=50$ for fixed $\lambda_{4}=200$ and $\lambda_{6}=-\frac{1000}{3}$, respectively. (b) The solid line is for $\lambda_{4}=0$, the dashed line is for $\lambda_{4}=-250$ and the dot-dashed line is for $\lambda_{4}=250$ for fixed $\lambda_{2}=-25$ and $\lambda_{6}=-\frac{1000}{3}$, respectively. (c) The solid line is for $\lambda_{6}=0$, the dashed line is for $\lambda_{6}=-\frac{2000}{3}$ and the dot-dashed line is for $\lambda_{6}=\frac{2000}{3}$ for fixed $\lambda_{2}=-25$ and $\lambda_{4}=200$, respectively.

Generally, if one works in the full back-reaction scenario, the parameters $\lambda_{2}, \lambda_{4}, \lambda_{6}$ will back-reacting the background geometric and also the equation of states. It could affect the baryon number susceptibilities $\chi_{n}^{B}$ even at $\mu=0$, since $\chi_{n}^{B}$ depends on the derivative with respect to $\mu$. In this way, one might fix $\lambda_{2}, \lambda_{4}, \lambda_{6}$ by fitting the lattice results at $\mu=0$. However, in this work, we neglect the back-reaction. Therefore, we can not fix these three parameters in this way. In figure 7 , we show the effects of $\lambda \mathrm{s}$ on chiral condensate in wide parameter regions, $-50 \leq \lambda_{2} \leq 50,-250 \leq \lambda_{4} \leq 250,-\frac{-2000}{3} \leq \lambda_{6} \leq \frac{2000}{3}$. From figure 7 , we could see that the parameters $\lambda$ is the coupling strength of chiral condensate with chemical potential and the sensitivity of $\lambda$ is presented. It is found that the effect of $\lambda_{2}$ is dominated, comparing with high order coupling term $\lambda_{4}$ and $\lambda_{6}$. The positive/negative sign in front of $\lambda$ will increase/decrease the transition temperature at the same chemical potential, which also means the location of CEP will appear at lower/higher chemical potential in $T-\mu$ plane. Without the coupling of chiral condensate with chemical potential, the deconfinement transition and chiral phase transition line will almost overlap. Thus, 


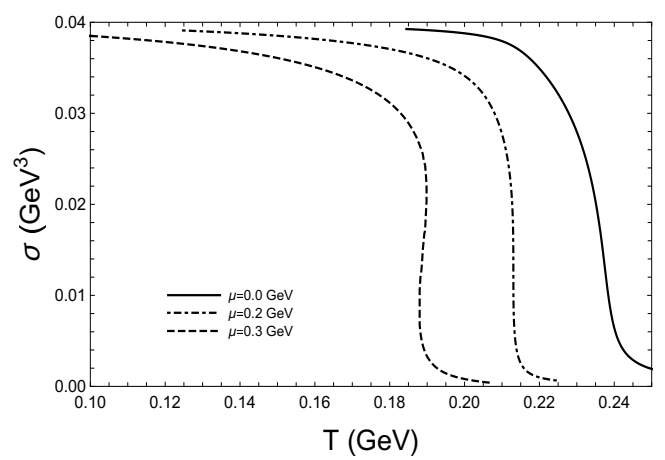

Figure 8. The chiral condensate $\sigma$ as a function of the temperature $\mathrm{T}$ for three different quark chemical potentials $\mu$. The solid line is for $\mu=0 \mathrm{GeV}$, the dashed-dotted line is for $\mu=0.2 \mathrm{GeV}$ and the dashed line is for $\mu=0.3 \mathrm{GeV}$, respectively. The crossover becomes first order transition at high chemical potential.

we choose these certain set of $\lambda$ to show the qualitative behavior of coupling strength in this model. Therefore, in the later calculation we will take a specific group of parameters $\lambda_{2}=-25, \lambda_{4}=200, \lambda_{6}=-\frac{1000}{3}$ to show the qualitative results and the approximate quantitative results to get a close CEP position of PNJL model. But we emphasize that the exact value of this parameters could be fixed in a full back-reaction scenario and the position of CEP could be predicted in that model, which will be left for the future study.

\subsection{Interplay of chiral and deconfinement phase transition: quarkyonic phase}

According to the above discussion, finally, we will take the parameters $\lambda_{0}=0, \lambda_{2}=-25$, $\lambda_{4}=200, \lambda_{6}=-\frac{1000}{3}, v_{4}=2, v_{6}=100$ in our calculation. Taking the physical quark mass $m_{q}=5 \mathrm{MeV}$, we could solve out chiral condensate $\sigma$ as function of $T$ and $\mu$. We find that at small chemical potential, the phase transition is of crossover type, like the dashed line for $\mu=0$ in figure 8. At large chemical potential, it is found that the phase transition turns to first order one, with multi-valued region appears, like the solid line for $\mu=0.3 \mathrm{GeV}$ in figure 8 . In between the two cases, there is a critical point, where the phase transition turns to second order type, like the dotdashed line with $\mu^{E}=0.21 \mathrm{GeV}$ in figure 8. One could define the second order transition temperature at $\frac{\partial \sigma}{\partial T}=\infty$, and we get $T^{E}=0.20 \mathrm{GeV}$. So, we get when $\mu<\mu^{E}$, the phase transition is crossover type while when $\mu>\mu^{E}$ it turns to be first order one. In between them, it is the critical end point $\left(T^{E}, \mu^{E}\right)=(0.20,0.21) \mathrm{GeV}$ or $\left(T^{E}, \mu_{B}^{E}\right)=(0.20,0.63) \mathrm{GeV}$. One could see that the critical baryon chemical potential at CEP in the quenched dynamical holographic QCD model is in good agreement with that in the realistic PNJL model [110]. Finally, varying chemical potential and extracting the corresponding critical temperature, one could draw the phase transition line for chiral phase transition in the black lines in figure 9 .

We summarize the chiral and deconfinement phase transitions in the $(T, \mu)$ plane in figure 9. Both the deconfinement and chiral phase transitions are realized in the quenched dynamical holographic QCD model. The deconfinement phase transition is always a crossover and it shows weak dependence on the quark chemical potential, and the chiral phase transition is a crossover at low chemical potential and turns to a first order phase transition at 


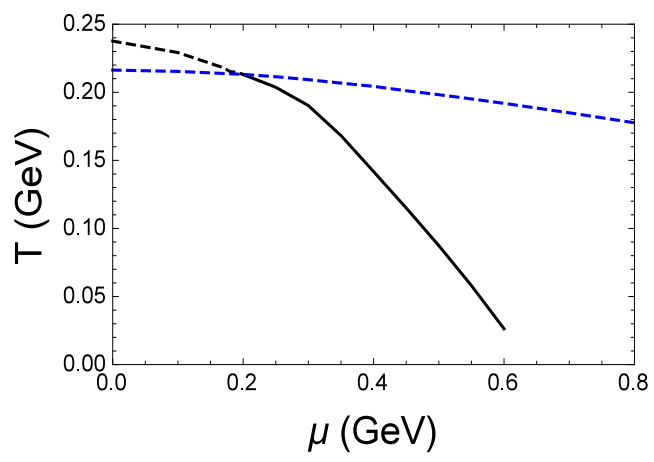

Figure 9. The $(T, \mu)$ phase diagram for chiral and deconfinement phase transitions. The blue dashed line is for deconfinement phase transition and the black line is for the chiral phase transition, respectively.

high chemical potential, and a CEP shows up at $\left(T^{E}, \mu^{E}\right)=(0.20,0.21) \mathrm{GeV}$ on the chiral phase transition line. The chiral phase transition has much stronger dependence on the quark chemical potential than the deconfinement phase transition, thus one can observe the quarkyonic phase showing up in the region of large chemical potential. This phase diagram is in agreement with that in the PNJL model [10, 110, 121].

\section{Conclusion}

In this work, we investigate both the chiral and deconfinement phase transitions at finite temperature and chemical potential in the quenched dynamical holographic QCD model. In this quenched dynamical holographic QCD model, the dilaton background describes the gluodynamics and the flavor/meson background describes the chiral dynamics, respectively. To extend the quenched dynamical holographic QCD model to finite chemical potential, the quark chemical potential is introduced by a U(1) field in the Einstein-Dilaton-Maxwell framework. The chemical potential dependence of the dilaton/gluodynamics is fixed by higher order baryon number fluctuations especially the kurtosis of baryon number fluctuations. For the matter sector, we introduce a sextic term in the scalar potential to realize the first order phase transition at high chemical potential.

The chiral and deconfinement phase transitions in the $(T, \mu)$ plane is qualitatively consistent with that in the PNJL model. The deconfinement phase transition is always a crossover in the $(T, \mu)$ plane and it shows weak dependence on the quark chemical potential. The chiral phase transition is a crossover at low chemical potential and turns to be a first order phase transition at high chemical potential with a CEP showing up at $\left(T^{E}, \mu^{E}\right)=(0.20,0.21) \mathrm{GeV}$. The chiral phase transition has much stronger dependence on the quark chemical potential, therefore a quarkyonic phase with chiral symmetry restoration but still in confinement showing up in the region of large chemical potential. It is not surprising that the quenched dynamical holographic QCD model shows qualitatively consistent result with the PNJL model, because the PNJL model is also a model of quenched gluon background plus quark dynamics. 
Naturally, we will consider the back reaction of the matter part action on the quenched gluodynamic background. Due to the complexity of numerical calculations of the coupling of two actions, we will try to solve the phase structure of the full dynamical holographic QCD model in the future.

\section{Acknowledgments}

D.L. is supported by the NSFC under Grant Nos. 11805084 and 11647141, D.F.H is supported by the Ministry of Science and Technology of China (MSTC) under the 973 Project No. 2015CB856904(4) and by the NSFC under Grant No. 11735007, and M.H. is supported in part by the NSFC under Grant Nos. 11725523, 11735007, 11261130311 (CRC 110 by DFG and NSFC), Chinese Academy of Sciences under Grant No. XDPB09, and the start-up funding from University of Chinese Academy of Sciences (UCAS).

Open Access. This article is distributed under the terms of the Creative Commons Attribution License (CC-BY 4.0), which permits any use, distribution and reproduction in any medium, provided the original author(s) and source are credited.

\section{References}

[1] A.M. Polyakov, Thermal Properties of Gauge Fields and Quark Liberation, Phys. Lett. 72B (1978) 477 [INSPIRE].

[2] G. 't Hooft, On the Phase Transition Towards Permanent Quark Confinement, Nucl. Phys. B 138 (1978) 1 [INSPIRE].

[3] A. Casher, Chiral Symmetry Breaking in Quark Confining Theories, Phys. Lett. 83B (1979) 395 [INSPIRE].

[4] T. Banks and A. Casher, Chiral Symmetry Breaking in Confining Theories, Nucl. Phys. B 169 (1980) 103 [INSPIRE].

[5] Y. Hatta and K. Fukushima, Linking the chiral and deconfinement phase transitions, Phys. Rev. D 69 (2004) 097502 [hep-ph/0307068] [INSPIRE].

[6] A. Mócsy, F. Sannino and K. Tuominen, Confinement versus chiral symmetry, Phys. Rev. Lett. 92 (2004) 182302 [hep-ph/0308135] [INSPIRE].

[7] J. Braun, H. Gies and J.M. Pawlowski, Quark Confinement from Color Confinement, Phys. Lett. B 684 (2010) 262 [arXiv:0708.2413] [InSPIRE].

[8] L. McLerran and R.D. Pisarski, Phases of cold, dense quarks at large $N_{c}$, Nucl. Phys. A 796 (2007) 83 [arXiv:0706.2191] [INSPIRE].

[9] Y. Hidaka, L.D. McLerran and R.D. Pisarski, Baryons and the phase diagram for a large number of colors and flavors, Nucl. Phys. A 808 (2008) 117 [arXiv:0803.0279] [InSPIRE].

[10] L. McLerran, K. Redlich and C. Sasaki, Quarkyonic Matter and Chiral Symmetry Breaking, Nucl. Phys. A 824 (2009) 86 [arXiv:0812.3585] [InSPIRE].

[11] Y. Aoki, Z. Fodor, S.D. Katz and K.K. Szabo, The QCD transition temperature: Results with physical masses in the continuum limit, Phys. Lett. B 643 (2006) 46 [hep-lat/0609068] [INSPIRE]. 
[12] C. Schmidt, Lattice QCD at finite density, PoS (LAT2006) 021 (2006) [hep-lat/0610116] [INSPIRE].

[13] O. Philipsen, The QCD phase diagram at zero and small baryon density, PoS(LAT2005) 016 (2006) [hep-lat/0510077] [INSPIRE].

[14] U.M. Heller, Recent progress in finite temperature lattice QCD, PoS (LAT2006) 011 (2006) [hep-lat/0610114] [INSPIRE].

[15] R. Alkofer and L. von Smekal, The Infrared behavior of QCD Green's functions: Confinement dynamical symmetry breaking and hadrons as relativistic bound states, Phys. Rept. 353 (2001) 281 [hep-ph/0007355] [INSPIRE].

[16] A. Bashir et al., Collective perspective on advances in Dyson-Schwinger Equation QCD, Commun. Theor. Phys. 58 (2012) 79 [arXiv:1201.3366] [INSPIRE].

[17] C. Wetterich, Exact evolution equation for the effective potential, Phys. Lett. B 301 (1993) 90 [arXiv: 1710.05815] [INSPIRE].

[18] J.M. Pawlowski, Aspects of the functional renormalisation group, Annals Phys. 322 (2007) 2831 [hep-th/0512261] [INSPIRE].

[19] H. Gies, Introduction to the functional $R G$ and applications to gauge theories, Lect. Notes Phys. 852 (2012) 287 [hep-ph/0611146] [INSPIRE].

[20] Y. Nambu and G. Jona-Lasinio, Dynamical model of elementary particles based on an analogy with superconductivity. II, Phys. Rev. 124 (1961) 246 [INSPIRE].

[21] S.P. Klevansky, The Nambu-Jona-Lasinio model of quantum chromodynamics, Rev. Mod. Phys. 64 (1992) 649 [INSPIRE].

[22] P.N. Meisinger and M.C. Ogilvie, Chiral symmetry restoration and $Z(N)$ symmetry, Phys. Lett. B 379 (1996) 163 [hep-lat/9512011] [INSPIRE].

[23] K. Fukushima, Chiral effective model with the Polyakov loop, Phys. Lett. B 591 (2004) 277 [hep-ph/0310121] [INSPIRE].

[24] C. Ratti, M.A. Thaler and W. Weise, Phases of QCD: Lattice thermodynamics and a field theoretical model, Phys. Rev. D 73 (2006) 014019 [hep-ph/0506234] [INSPIRE].

[25] S. Roessner, C. Ratti and W. Weise, Polyakov loop, diquarks and the two-flavour phase diagram, Phys. Rev. D 75 (2007) 034007 [hep-ph/0609281] [INSPIRE].

[26] S.K. Ghosh, T.K. Mukherjee, M.G. Mustafa and R. Ray, Susceptibilities and speed of sound from PNJL model, Phys. Rev. D 73 (2006) 114007 [hep-ph/0603050] [INSPIRE].

[27] B.-J. Schaefer, J.M. Pawlowski and J. Wambach, The Phase Structure of the Polyakov-Quark-Meson Model, Phys. Rev. D 76 (2007) 074023 [arXiv:0704.3234] [INSPIRE].

[28] C. Ratti, S. Roessner and W. Weise, Quark number susceptibilities: Lattice QCD versus PNJL model, Phys. Lett. B 649 (2007) 57 [hep-ph/0701091] [INSPIRE].

[29] C. Sasaki, B. Friman and K. Redlich, Susceptibilities and the Phase Structure of a Chiral Model with Polyakov Loops, Phys. Rev. D 75 (2007) 074013 [hep-ph/0611147] [INSPIRE].

[30] C. Sasaki, B. Friman and K. Redlich, Quark Number Fluctuations in a Chiral Model at Finite Baryon Chemical Potential, Phys. Rev. D 75 (2007) 054026 [hep-ph/0611143] [INSPIRE]. 
[31] E. Megias, E. Ruiz Arriola and L.L. Salcedo, Chiral Lagrangian at finite temperature from the Polyakov-Chiral Quark Model, Phys. Rev. D 74 (2006) 114014 [hep-ph/0607338] [INSPIRE].

[32] E. Megias, E. Ruiz Arriola and L.L. Salcedo, Polyakov loop in chiral quark models at finite temperature, Phys. Rev. D 74 (2006) 065005 [hep-ph/0412308] [INSPIRE].

[33] Z. Zhang and Y.-X. Liu, Coupling of pion condensate, chiral condensate and Polyakov loop in an extended NJLS model, Phys. Rev. C 75 (2007) 064910 [hep-ph/0610221] [INSPIRE].

[34] Y. Sakai, K. Kashiwa, H. Kouno and M. Yahiro, Polyakov loop extended NJLS model with imaginary chemical potential, Phys. Rev. D 77 (2008) 051901 [arXiv:0801. 0034] [INSPIRE].

[35] M. Ciminale, G. Nardulli, M. Ruggieri and R. Gatto, Polyakov loop and the color-flavor locked phase of quantum chromodynamics, Phys. Lett. B 657 (2007) 64 [arXiv:0706.4215] [INSPIRE].

[36] W.-j. Fu, Z. Zhang and Y.-x. Liu, 2+1 flavor Polyakov-Nambu-Jona-Lasinio model at finite temperature and nonzero chemical potential, Phys. Rev. D 77 (2008) 014006 [arXiv: 0711.0154] [INSPIRE].

[37] H. Hansen, W.M. Alberico, A. Beraudo, A. Molinari, M. Nardi and C. Ratti, Mesonic correlation functions at finite temperature and density in the Nambu-Jona-Lasinio model with a Polyakov loop, Phys. Rev. D 75 (2007) 065004 [hep-ph/0609116] [InSPIRE].

[38] G.A. Contrera, D. Gomez Dumm and N.N. Scoccola, Nonlocal SU(3) chiral quark models at finite temperature: The Role of the Polyakov loop, Phys. Lett. B 661 (2008) 113 [arXiv:0711.0139] [INSPIRE].

[39] J.M. Maldacena, The Large $N$ limit of superconformal field theories and supergravity, Int. J. Theor. Phys. 38 (1999) 1113 [hep-th/9711200] [InSPIRE].

[40] S.S. Gubser, I.R. Klebanov and A.M. Polyakov, Gauge theory correlators from noncritical string theory, Phys. Lett. B 428 (1998) 105 [hep-th/9802109] [INSPIRE].

[41] E. Witten, Anti-de Sitter space and holography, Adv. Theor. Math. Phys. 2 (1998) 253 [hep-th/9802150] [INSPIRE].

[42] J. Erlich, E. Katz, D.T. Son and M.A. Stephanov, QCD and a holographic model of hadrons, Phys. Rev. Lett. 95 (2005) 261602 [hep-ph/0501128] [INSPIRE].

[43] G.F. de Teramond and S.J. Brodsky, Hadronic spectrum of a holographic dual of $Q C D$, Phys. Rev. Lett. 94 (2005) 201601 [hep-th/0501022] [INSPIRE].

[44] L. Da Rold and A. Pomarol, Chiral symmetry breaking from five dimensional spaces, Nucl. Phys. B 721 (2005) 79 [hep-ph/0501218] [INSPIRE].

[45] J. Babington, J. Erdmenger, N.J. Evans, Z. Guralnik and I. Kirsch, Chiral symmetry breaking and pions in nonsupersymmetric gauge / gravity duals, Phys. Rev. D 69 (2004) 066007 [hep-th/0306018] [INSPIRE].

[46] M. Kruczenski, D. Mateos, R.C. Myers and D.J. Winters, Towards a holographic dual of large $N_{c} Q C D$, JHEP 05 (2004) 041 [hep-th/0311270] [INSPIRE].

[47] T. Sakai and S. Sugimoto, Low energy hadron physics in holographic QCD, Prog. Theor. Phys. 113 (2005) 843 [hep-th/0412141] [INSPIRE].

[48] T. Sakai and S. Sugimoto, More on a holographic dual of QCD, Prog. Theor. Phys. 114 (2005) 1083 [hep-th/0507073] [INSPIRE]. 
[49] C. Csáki and M. Reece, Toward a systematic holographic QCD: A Braneless approach, JHEP 05 (2007) 062 [hep-ph/0608266] [INSPIRE].

[50] S. He, M. Huang, Q.-S. Yan and Y. Yang, Confront Holographic QCD with Regge Trajectories, Eur. Phys. J. C 66 (2010) 187 [arXiv:0710.0988] [InSPIRE].

[51] T. Gherghetta, J.I. Kapusta and T.M. Kelley, Chiral symmetry breaking in the soft-wall AdS/QCD model, Phys. Rev. D 79 (2009) 076003 [arXiv:0902.1998] [INSPIRE].

[52] T.M. Kelley, S.P. Bartz and J.I. Kapusta, Pseudoscalar Mass Spectrum in a Soft-Wall Model of AdS/QCD, Phys. Rev. D 83 (2011) 016002 [arXiv: 1009.3009] [inSPIRE].

[53] Y.-Q. Sui, Y.-L. Wu, Z.-F. Xie and Y.-B. Yang, Prediction for the Mass Spectra of Resonance Mesons in the Soft-Wall AdS/QCD with a Modified 5D Metric, Phys. Rev. D 81 (2010) 014024 [arXiv:0909.3887] [INSPIRE].

[54] Y.-Q. Sui, Y.-L. Wu and Y.-B. Yang, Predictive AdS/QCD Model for Mass Spectra of Mesons with Three Flavors, Phys. Rev. D 83 (2011) 065030 [arXiv:1012.3518] [InSPIRE].

[55] D. Li, M. Huang and Q.-S. Yan, A dynamical soft-wall holographic QCD model for chiral symmetry breaking and linear confinement, Eur. Phys. J. C 73 (2013) 2615 [arXiv:1206.2824] [INSPIRE].

[56] D. Li and M. Huang, Dynamical holographic QCD model for glueball and light meson spectra, JHEP 11 (2013) 088 [arXiv: 1303.6929] [INSPIRE].

[57] Y. Chen and M. Huang, Two-gluon and trigluon glueballs from dynamical holography QCD, Chin. Phys. C 40 (2016) 123101 [arXiv:1511.07018] [InSPIRE].

[58] E.V. Shuryak, What RHIC experiments and theory tell us about properties of quark-gluon plasma?, Nucl. Phys. A 750 (2005) 64 [hep-ph/0405066] [INSPIRE].

[59] M.J. Tannenbaum, Recent results in relativistic heavy ion collisions: From 'a new state of matter' to 'the perfect fluid', Rept. Prog. Phys. 69 (2006) 2005 [nucl-ex/0603003] [INSPIRE].

[60] G. Policastro, D.T. Son and A.O. Starinets, The Shear viscosity of strongly coupled $N=4$ supersymmetric Yang-Mills plasma, Phys. Rev. Lett. 87 (2001) 081601 [hep-th/0104066] [INSPIRE].

[61] R.-G. Cai, Z.-Y. Nie, N. Ohta and Y.-W. Sun, Shear Viscosity from Gauss-Bonnet Gravity with a Dilaton Coupling, Phys. Rev. D 79 (2009) 066004 [arXiv:0901.1421] [InSPIRE].

[62] R.-G. Cai, Z.-Y. Nie and Y.-W. Sun, Shear Viscosity from Effective Couplings of Gravitons, Phys. Rev. D 78 (2008) 126007 [arXiv:0811.1665] [INSPIRE].

[63] S.-J. Sin and I. Zahed, Holography of radiation and jet quenching, Phys. Lett. B 608 (2005) 265 [hep-th/0407215] [INSPIRE].

[64] E. Shuryak, S.-J. Sin and I. Zahed, A Gravity dual of RHIC collisions, J. Korean Phys. Soc. 50 (2007) 384 [hep-th/0511199] [INSPIRE].

[65] R.A. Janik and R.B. Peschanski, Asymptotic perfect fluid dynamics as a consequence of AdS/CFT, Phys. Rev. D 73 (2006) 045013 [hep-th/0512162] [INSPIRE].

[66] S. Nakamura and S.-J. Sin, A Holographic dual of hydrodynamics, JHEP 09 (2006) 020 [hep-th/0607123] [INSPIRE].

[67] S.-J. Sin, S. Nakamura and S.P. Kim, Elliptic Flow, Kasner Universe and Holographic Dual of RHIC Fireball, JHEP 12 (2006) 075 [hep-th/0610113] [INSPIRE]. 
[68] C.P. Herzog, A. Karch, P. Kovtun, C. Kozcaz and L.G. Yaffe, Energy loss of a heavy quark moving through $N=4$ supersymmetric Yang-Mills plasma, JHEP 07 (2006) 013 [hep-th/0605158] [INSPIRE].

[69] S.S. Gubser, Drag force in AdS/CFT, Phys. Rev. D 74 (2006) 126005 [hep-th/0605182] [INSPIRE].

[70] Z.-q. Zhang, D.-f. Hou and H.-c. Ren, The finite 't Hooft coupling correction on jet quenching parameter in a $\mathcal{N}=4$ Super Yang-Mills Plasma, JHEP 01 (2013) 032 [arXiv: 1210.5187] [INSPIRE].

[71] D. Li, J. Liao and M. Huang, Enhancement of jet quenching around phase transition: result from the dynamical holographic model, Phys. Rev. D 89 (2014) 126006 [arXiv:1401.2035] [INSPIRE].

[72] D. Li, S. He and M. Huang, Temperature dependent transport coefficients in a dynamical holographic QCD model, JHEP 06 (2015) 046 [arXiv:1411.5332] [INSPIRE].

[73] O. Aharony, S.S. Gubser, J.M. Maldacena, H. Ooguri and Y. Oz, Large $N$ field theories, string theory and gravity, Phys. Rept. 323 (2000) 183 [hep-th/9905111] [INSPIRE].

[74] J. Erdmenger, N. Evans, I. Kirsch and E. Threlfall, Mesons in Gauge/Gravity Duals - A Review, Eur. Phys. J. A 35 (2008) 81 [arXiv:0711.4467] [InSPIRE].

[75] S.J. Brodsky, G.F. de Teramond, H.G. Dosch and J. Erlich, Light-Front Holographic QCD and Emerging Confinement, Phys. Rept. 584 (2015) 1 [arXiv:1407.8131] [INSPIRE].

[76] Y. Kim, I.J. Shin and T. Tsukioka, Holographic QCD: Past, Present and Future, Prog. Part. Nucl. Phys. 68 (2013) 55 [arXiv:1205.4852] [InSPIRE].

[77] A. Adams, L.D. Carr, T. Schäfer, P. Steinberg and J.E. Thomas, Strongly Correlated Quantum Fluids: Ultracold Quantum Gases, Quantum Chromodynamic Plasmas and Holographic Duality, New J. Phys. 14 (2012) 115009 [arXiv:1205.5180] [InSPIRE].

[78] C.P. Herzog, A Holographic Prediction of the Deconfinement Temperature, Phys. Rev. Lett. 98 (2007) 091601 [hep-th/0608151] [INSPIRE].

[79] C.A. Ballon Bayona, H. Boschi-Filho, N.R.F. Braga and L.A. Pando Zayas, On a Holographic Model for Confinement/Deconfinement, Phys. Rev. D 77 (2008) 046002 [arXiv:0705.1529] [INSPIRE].

[80] R.-G. Cai and J.P. Shock, Holographic confinement/deconfinement phase transitions of $A d S / Q C D$ in curved spaces, JHEP 08 (2007) 095 [arXiv:0705.3388] [INSPIRE].

[81] R.-G. Cai, S. Chakrabortty, S. He and L. Li, Some aspects of QGP phase in a hQCD model, JHEP 02 (2013) 068 [arXiv:1209.4512] [InSPIRE].

[82] Y. Kim, B.-H. Lee, S. Nam, C. Park and S.-J. Sin, Deconfinement phase transition in holographic QCD with matter, Phys. Rev. D 76 (2007) 086003 [arXiv:0706.2525] [INSPIRE].

[83] O. Andreev, Renormalized Polyakov Loop in the Deconfined Phase of $\mathrm{SU}(N)$ Gauge Theory and Gauge/String Duality, Phys. Rev. Lett. 102 (2009) 212001 [arXiv:0903.4375] [INSPIRE].

[84] P. Colangelo, F. Giannuzzi and S. Nicotri, Holography, Heavy-Quark Free Energy and the QCD Phase Diagram, Phys. Rev. D 83 (2011) 035015 [arXiv:1008.3116] [INSPIRE].

[85] S.S. Gubser, A. Nellore, S.S. Pufu and F.D. Rocha, Thermodynamics and bulk viscosity of approximate black hole duals to finite temperature quantum chromodynamics, Phys. Rev. Lett. 101 (2008) 131601 [arXiv:0804.1950] [INSPIRE]. 
[86] S.S. Gubser and A. Nellore, Mimicking the QCD equation of state with a dual black hole, Phys. Rev. D 78 (2008) 086007 [arXiv:0804.0434] [InSPIRE].

[87] S.S. Gubser, S.S. Pufu and F.D. Rocha, Bulk viscosity of strongly coupled plasmas with holographic duals, JHEP 08 (2008) 085 [arXiv: 0806. 0407] [inSPIRE].

[88] U. Gürsoy, E. Kiritsis, L. Mazzanti and F. Nitti, Deconfinement and Gluon Plasma Dynamics in Improved Holographic QCD, Phys. Rev. Lett. 101 (2008) 181601 [arXiv:0804.0899] [INSPIRE].

[89] U. Gürsoy and E. Kiritsis, Exploring improved holographic theories for QCD: Part I, JHEP 02 (2008) 032 [arXiv: 0707.1324] [INSPIRE].

[90] U. Gürsoy, E. Kiritsis and F. Nitti, Exploring improved holographic theories for QCD: Part II, JHEP 02 (2008) 019 [arXiv:0707.1349] [INSPIRE].

[91] U. Gürsoy, E. Kiritsis, L. Mazzanti and F. Nitti, Holography and Thermodynamics of $5 D$ Dilaton-gravity, JHEP 05 (2009) 033 [arXiv:0812.0792] [INSPIRE].

[92] R. Yaresko and B. Kampfer, Equation of State and Viscosities from a Gravity Dual of the Gluon Plasma, Phys. Lett. B $\mathbf{7 4 7}$ (2015) 36 [arXiv:1306. 0214] [InSPIRE].

[93] D. Li, S. He, M. Huang and Q.-S. Yan, Thermodynamics of deformed AdS $S_{5}$ model with a positive/negative quadratic correction in graviton-dilaton system, JHEP 09 (2011) 041 [arXiv: 1103.5389] [INSPIRE].

[94] R.-G. Cai, S. He and D. Li, A hQCD model and its phase diagram in Einstein-Maxwell-Dilaton system, JHEP 03 (2012) 033 [arXiv:1201.0820] [INSPIRE].

[95] S. He, S.-Y. Wu, Y. Yang and P.-H. Yuan, Phase Structure in a Dynamical Soft-Wall Holographic QCD Model, JHEP 04 (2013) 093 [arXiv: 1301.0385] [INSPIRE].

[96] Y. Yang and P.-H. Yuan, A Refined Holographic QCD Model and QCD Phase Structure, JHEP 11 (2014) 149 [arXiv:1406.1865] [INSPIRE].

[97] F. Zuo, Thermal power terms in the Einstein-dilaton system, JHEP 06 (2014) 143 [arXiv: 1404.4512] [INSPIRE].

[98] S.S. Afonin and A.D. Katanaeva, Holographic Estimates of the Deconfinement Temperature, Eur. Phys. J. C 74 (2014) 3124 [arXiv: 1408.6935] [InSPIRE].

[99] F. Bruckmann, G. Endrodi and T.G. Kovacs, Inverse magnetic catalysis and the Polyakov loop, JHEP 04 (2013) 112 [arXiv:1303.3972] [INSPIRE].

[100] R. Rougemont, R. Critelli and J. Noronha, Holographic calculation of the QCD crossover temperature in a magnetic field, Phys. Rev. D 93 (2016) 045013 [arXiv:1505.07894] [INSPIRE].

[101] A. Karch, E. Katz, D.T. Son and M.A. Stephanov, Linear confinement and AdS/QCD, Phys. Rev. D 74 (2006) 015005 [hep-ph/0602229] [INSPIRE].

[102] K. Chelabi, Z. Fang, M. Huang, D. Li and Y.-L. Wu, Realization of chiral symmetry breaking and restoration in holographic QCD, Phys. Rev. D 93 (2016) 101901 [arXiv: 1511.02721] [INSPIRE].

[103] K. Chelabi, Z. Fang, M. Huang, D. Li and Y.-L. Wu, Chiral Phase Transition in the Soft-Wall Model of AdS/QCD, JHEP 04 (2016) 036 [arXiv: 1512.06493] [INSPIRE].

[104] Z. Fang, S. He and D. Li, Chiral and Deconfining Phase Transitions from Holographic QCD Study, Nucl. Phys. B 907 (2016) 187 [arXiv:1512.04062] [INSPIRE]. 
[105] N. Evans, C. Miller and M. Scott, Inverse Magnetic Catalysis in Bottom-Up Holographic QCD, Phys. Rev. D 94 (2016) 074034 [arXiv: 1604.06307] [inSPIRE].

[106] N. Evans, A. Gebauer, K.-Y. Kim and M. Magou, Holographic Description of the Phase Diagram of a Chiral Symmetry Breaking Gauge Theory, JHEP 03 (2010) 132 [arXiv: 1002.1885] [INSPIRE].

[107] N. Evans, K.-Y. Kim, M. Magou, Y. Seo and S.-J. Sin, The Baryonic Phase in Holographic Descriptions of the QCD Phase Diagram, JHEP 09 (2012) 045 [arXiv:1204.5640] [INSPIRE].

[108] N. Evans, A. Gebauer, M. Magou and K.-Y. Kim, Towards a Holographic Model of the QCD Phase Diagram, J. Phys. G 39 (2012) 054005 [arXiv:1109.2633] [InSPIRE].

[109] Y. Sakai, H. Kouno, T. Sasaki and M. Yahiro, The quarkyonic phase and the $Z_{N_{c}}$ symmetry, Phys. Lett. B 718 (2012) 130 [arXiv: 1204.0228] [INSPIRE].

[110] Z. Li, K. Xu, X. Wang and M. Huang, The kurtosis of net baryon number fluctuations from a realistic Polyakov-Nambu-Jona-Lasinio model along the experimental freeze-out line, Eur. Phys. J. C 79 (2019) 245 [arXiv:1801.09215] [InSPIRE].

[111] Z. Li, Y. Chen, D. Li and M. Huang, Locating the QCD critical end point through the peaked baryon number susceptibilities along the freeze-out line, Chin. Phys. C 42 (2018) 013103 [arXiv: 1706. 02238] [INSPIRE].

[112] A. Bazavov et al., The QCD Equation of State to $\mathcal{O}\left(\mu_{B}^{6}\right)$ from Lattice QCD, Phys. Rev. D 95 (2017) 054504 [arXiv: 1701.04325] [INSPIRE].

[113] M. Huang and D. Li, Dynamical holographic QCD model: resembling renormalization group from ultraviolet to infrared, Springer Proc. Phys. 170 (2016) 367 [arXiv:1311.0593] [INSPIRE].

[114] D. Li and M. Huang, Dynamical holographic QCD model, EPJ Web Conf. 80 (2014) 00011 [arXiv: 1409.8432] [INSPIRE].

[115] G. 't Hooft, Dimensional reduction in quantum gravity, Conf. Proc. C 930308 (1993) 284 [gr-qc/9310026] [INSPIRE].

[116] L. Susskind, The World as a hologram, J. Math. Phys. 36 (1995) 6377 [hep-th/9409089] [INSPIRE].

[117] TMFT collaboration, Equation of state of quark-gluon matter from lattice QCD with two flavors of twisted mass Wilson fermions, Phys. Rev. D 91 (2015) 074504 [arXiv: 1412.6748] [INSPIRE].

[118] TMFT collaboration, Thermal QCD transition with two flavors of twisted mass fermions, Phys. Rev. D 87 (2013) 074508 [arXiv:1102.4530] [INSPIRE].

[119] A. Cherman, T.D. Cohen and E.S. Werbos, The Chiral condensate in holographic models of QCD, Phys. Rev. C 79 (2009) 045203 [arXiv: 0804.1096] [inSPIRE].

[120] J. Chen, S. He, M. Huang and D. Li, Critical exponents of finite temperature chiral phase transition in soft-wall AdS/QCD models, JHEP 01 (2019) 165 [arXiv:1810.07019] [INSPIRE].

[121] H. Abuki, R. Anglani, R. Gatto, G. Nardulli and M. Ruggieri, Chiral crossover, deconfinement and quarkyonic matter within a Nambu-Jona Lasinio model with the Polyakov loop, Phys. Rev. D 78 (2008) 034034 [arXiv:0805.1509] [INSPIRE]. 